\title{
Novel Method of Evaluating Liver Stiffness Using Transient Elastography to Evaluate Perioperative Status in Severe Heart Failure
}

\author{
Hiroyuki Nishi, MD; Koichi Toda, MD; Shigeru Miyagawa, MD; Yasushi Yoshikawa, MD; \\ Satsuki Fukushima, MD; Masashi Kawamura, MD; Tetsuya Saito, MD; \\ Daisuke Yoshioka, MD; Takashi Daimon, PhD; Yoshiki Sawa, MD
}

\begin{abstract}
Background: The aim of the present study was to assess the efficacy of a non-invasive method, transient elastography (FibroScan), in measuring liver stiffness (LS), and whether LS can be used as a marker of cardiac - and hence perioperative - status.

Methods and Results: Perioperative LS was prospectively measured using a FibroScan in 30 patients (21 male; 42.2 \pm 13.3 years old) who underwent left ventricular assist device (LVAD) implantation. LS was checked pre- and postoperatively, then analyzed in regard to perioperative status. Preoperative LS was $13.3 \pm 13.0 \mathrm{kPa}$ (normal, $<5.5 \mathrm{kPa}$ ), and was abnormal in $77 \%$ of patients. Four required bilateral VAD. LS in patients with bilateral VAD tended to be higher than in LVAD patients $(25.1 \pm 22.7$ vs. $11.5 \pm 10.5 \mathrm{kPa}, \mathrm{P}=0.051)$. No patient with LS $\leq 7.0 \mathrm{kPa}$ required a right VAD. The incidence of major adverse events was lower in patients with $\mathrm{LS} \leq 12.5 \mathrm{kPa}(25 \% \mathrm{vs} .80 \%$, $\mathrm{P}<0.05)$. There were also no mortalities among patients with $\mathrm{LS} \leq 12.5 \mathrm{kPa}$.

Conclusions: LS was correlated with preoperative severity in patients with severe heart failure and reflected liver congestion, and may be useful to predict the requirement of right VAD, as well as postoperative complications in patients with LVAD implantation. This novel modality may be a useful non-invasive assessment method for management of severe heart failure. (Circ $J$ 2015; 79: 391-397)
\end{abstract}

Key Words: Heart failure; Liver; Ventricular assist device

I $\mathrm{t}$ is recognized that cardiac dysfunction may affect liver function, resulting in cardiac hepatopathy. ${ }^{1-5}$ Liver function abnormalities are frequently found in patients with severe heart failure who require mechanical support such as a left ventricular assist device (LVAD) ${ }^{6-11}$ Heart failure is a pathologic condition in which inadequate blood pumping reduces flow and leads to congestion of blood and fluids in other organs. Furthermore, increased pressure in the right side of the heart is followed by dilatation of the inferior vena cava and hepatic veins, which causes an enlarged and firm liver. Previous reports have highlighted the importance of either high central venous pressure (CVP) or reduced hepatic perfusion. ${ }^{8,12}$ Scant information is available, however, regarding the relationship between the heart and liver, although liver condition might reflect the severity of heart failure. Recently, a rapid, non-invasive, and reproducible approach was developed to assess liver fibrosis by measuring liver stiffness (LS) using transient elastography. ${ }^{13,14}$ This new modality is user friendly, and provides im- mediate results and good reproducibility, and it has been used in many studies to demonstrate the correlation of LS with liver fibrosis. ${ }^{14,15}$ More recent investigations, however, have noted that LS is also affected by liver congestion ${ }^{16}$ and may be directly influenced by CVP. ${ }^{17}$ Unfortunately, no non-invasive tools are available to appropriately evaluate the severity of heart failure. In the present study, we focused on the impact of heart failure and changes in volume status on LS, and hypothesized that LS measured with transient elastography reflects cardiac status and can be used as a marker of perioperative status after LVAD implantation.

The purpose of this study was to assess the efficacy of noninvasive transient elastography during perioperative management of severe heart failure patients to measure variation in LS, and its relationship with clinical outcome and laboratory data. We also sought to clarify the relationship between LS and backward failure (liver congestion) in patients with severe heart failure, and assess the efficacy of transient elastography as a

Received August 19, 2014; revised manuscript received November 4, 2014; accepted November 5, 2014; released online December 10, 2014 Time for primary review: 14 days

Department of Cardiovascular Surgery, Osaka University Graduate School of Medicine, Suita (H.N., K.T., S.M., Y.Y., S.F., M.K., T.S., D.Y., Y.S.); Department of Biostatistics, Hyogo College of Medicine, Nishinomiya (T.D.), Japan

Mailing address: Yoshiaki Sawa, MD, Department of Cardiovascular Surgery, Osaka University Graduate School of Medicine, 2-2 Yamada-Oka, Suita 565-0871, Japan. E-mail: sawa-p@ @urg1.osaka-u.med.ac.jp

ISSN-1346-9843 doi:10.1253/circj.CJ-14-0929

All rights are reserved to the Japanese Circulation Society. For permissions, please e-mail: cj@j-circ.or.jp 


\begin{tabular}{|c|c|}
\hline Characteristics & Mean $\pm S D$ or $n$ \\
\hline Age (years) & $42.2 \pm 13.3$ \\
\hline Body weight (kg) & $57.4 \pm 14.0$ \\
\hline Gender (M/F) & $21 / 9$ \\
\hline \multicolumn{2}{|l|}{ Etiology } \\
\hline Dilated cardiomyopathy & 20 \\
\hline $\begin{array}{l}\text { Hypertrophic cardiomyopathy } \\
\text { (dilated phase) }\end{array}$ & 3 \\
\hline Ischemic cardiomyopathy & 2 \\
\hline Secondary cardiomyopathy & 5 \\
\hline \multicolumn{2}{|l|}{ LVAD } \\
\hline HeartWare & 10 \\
\hline HeartMate II & 9 \\
\hline DuraHeart & 4 \\
\hline EVAHEART & 4 \\
\hline Others & 3 \\
\hline INTERMACS profile & $1: 3,2: 16,3: 10,4: 1$ \\
\hline Preoperative T-bil (mg/dl) & $1.4 \pm 2.2$ \\
\hline Preoperative AST (IU/L) & $35 \pm 33$ \\
\hline Preoperative ALT (IU/L) & $49 \pm 94$ \\
\hline Preoperative Cr (mg/dl) & $1.4 \pm 0.9$ \\
\hline Preoperative CRP (mg/dl) & $2.3 \pm 3.3$ \\
\hline Preoperative BNP (pg/ml) & $844 \pm 806$ \\
\hline Preoperative CVP (mmHg) & $8.8 \pm 6.9$ \\
\hline Preoperative sPAP $(\mathrm{mmHg})$ & $46.2 \pm 18.6$ \\
\hline Preoperative PCWP $(\mathrm{mmHg})$ & $25.0 \pm 7.9$ \\
\hline \multicolumn{2}{|l|}{ Echocardiography } \\
\hline LVDd (mm) & $71.7 \pm 12.5$ \\
\hline LVEF (\%) & $21.7 \pm 7.4$ \\
\hline
\end{tabular}

ALT, alanine aminotransferase; AST, aspartate aminotransferase; $\mathrm{BNP}$, brain natriuretic peptide; $\mathrm{Cr}$, creatinine; $\mathrm{CRP}$, C-reactive protein; CVP, central venous pressure; INTERMACS, Interagency Registry for Mechanically Assisted Circulatory Support; LVAD, left ventricular assist device; LVDd, left ventricular diastolic dimension; LVEF, left ventricular ejection fraction; PCWP, pulmonary capillary wedge pressure; SPAP, systolic pulmonary arterial pressure; T-bil, total bilirubin.

method of evaluating severe heart failure.

\section{Methods}

\section{Patient Selection}

We prospectively investigated LS using transient elastography in 30 patients who underwent LVAD implantation at Osaka University Hospital between 2011 and 2013. Exclusion criteria included history of alcohol abuse, known chronic liver disease with an etiology other than heart failure, positivity for hepatitis C antibody or hepatitis B surface antigen reactivity, and obesity with a body mass index $>35 \mathrm{~kg} / \mathrm{m}^{2}$. Prior to surgery, all patients underwent routine blood test, including liver function tests such as aspartate aminotransferase (AST), alanine aminotransferase (ALT), and total bilirubin (T-bil). Cardiac function tests were also performed including brain natriuretic peptide (BNP), as well as echocardiography with determination of left ventricular ejection fraction (LVEF) and ventricular size, cardiac catheterization with hemodynamic variables such as right atrial pressure as an indicator of CVP, systolic pulmonary pressure (sPAP), and pulmonary capillary wedge pressure (PCWP). Informed consent was obtained from all patients
A Patients with preoperative liver stiffness $\leq 7.0 \mathrm{kPa}$

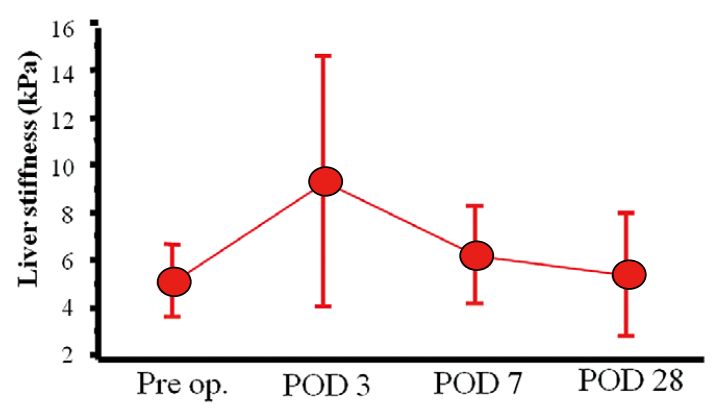

B Patients with preoperative liver stiffness $>7.0 \mathrm{kPa}$

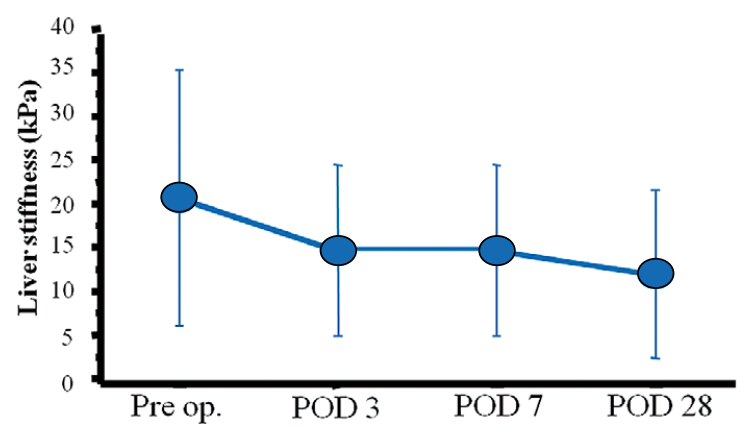

Figure 1. Changes in liver stiffness (LS) during the postoperative period in patients with $(\mathbf{A})$ preoperative $\mathrm{LS} \leq 7.0 \mathrm{kPa}$, and $(\mathrm{B})$ preoperative $\mathrm{LS}>7.0 \mathrm{kPa}$. (A) $\mathrm{LS}$ reached the maximum level on postoperative day (POD) 3 and was still high on POD 7, before returning to a normal level. (B) In contrast, LS gradually decreased during the postoperative period.

before surgery, and institutional review board approval for the study was also obtained. Baseline patient characteristics are listed in Table 1.

\section{Determination of LS Using Transient Elastography}

LS was determined using transient elastography (FibroScan; Echosens, Paris, France), as recently described in detail. ${ }^{18}$ The FibroScan consists of a 5-MHz US transducer probe mounted on the axis of a vibrator. Low-amplitude and low-frequency vibrations $(50 \mathrm{~Hz})$ induce an elastic shear wave that propagates through the underlying liver tissue at a velocity that is directly related to LS. In the present study the tip of the probe transducer was placed perpendicularly on the skin between the rib bones and the level of the right lobe of the liver with the patient lying in the dorsal decubitus position. The measurement depth was between 25 and $65 \mathrm{~mm}$ below the skin surface. In each evaluation, 10 measurements were performed with a success rate of at least $60 \%$ in each patient. The measurements are expressed in $\mathrm{kPa}$, and the median of 10 acquisitions was used. Based on previous studies and a recent meta-analysis, a cut-off of $<5.5 \mathrm{kPa}$ was considered to indicate normal. ${ }^{13}$

\section{Outcomes and Evaluation Factors}

We used 2 different endpoints, requirement of additional right heart support, defined as need for a right ventricular assist device (RVAD), and a major adverse event (MAE), defined as mortality, postoperative bleeding, cerebrovascular event, and infection within 30 days postoperatively. This definition of 
A

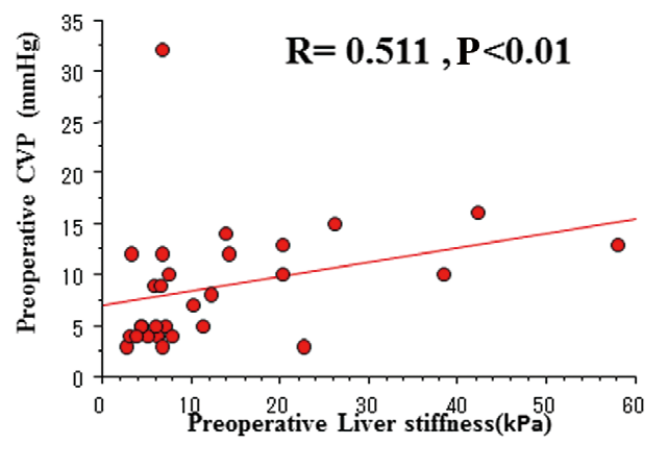

C

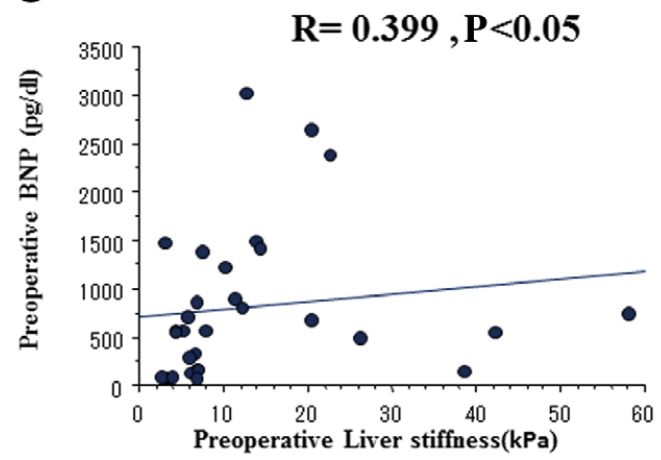

B

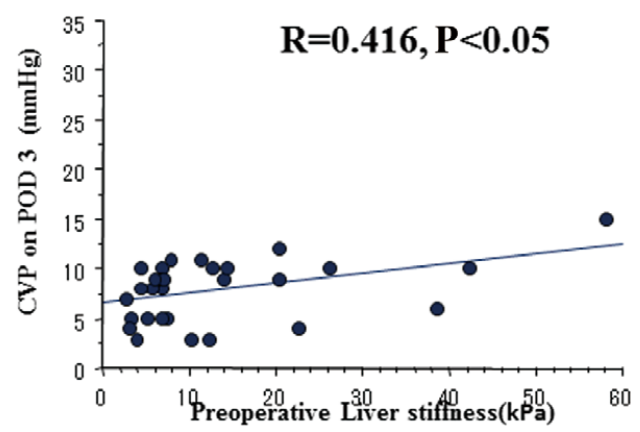

D

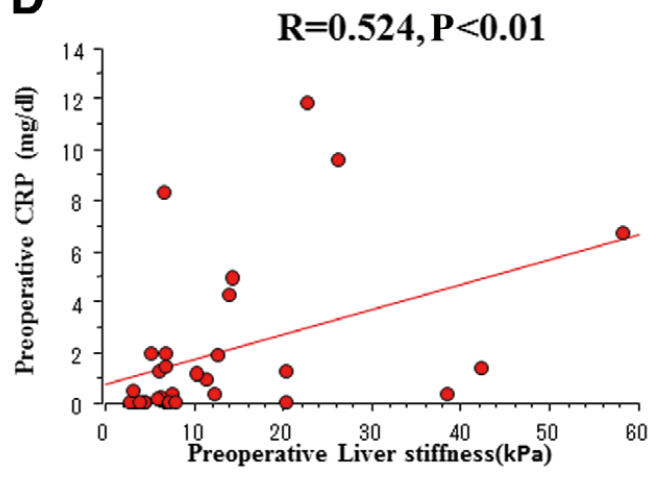

Figure 2. Correlations of preoperative liver stiffness with $(A)$ preoperative central venous pressure $(C V P ; r=0.515, n=30, P<0.01)$; (B) CVP on postoperative day (POD) $3(r=0.416, n=30, P<0.05)$; (C) preoperative b-type natriuretic peptide (BNP; $r=0.399$, $\mathrm{P}<0.05)$; and (D) preoperative C-reactive protein (CRP; $r=0.524, P<0.01)$.

MAE was used because these are serious complications that occur after LVAD implantation. We assessed the relationship between LS and each endpoint, and various perioperative parameters were evaluated to identify possible risk factors for an MAE.

The preoperative factors examined were age, sex, body weight, etiology, INTERMACS profile, blood parameters (preoperative AST, ALT, T-bil, creatinine [Cr], C-reactive protein [CRP]), and other preoperative comorbidities. Body weight and blood test results were obtained within 3 days prior to operation. Hemodynamic parameters were obtained from the most recent results prior to surgery. These factors were analyzed to identify possible risk factors on univariate analysis. Postoperative LS was obtained on postoperative day (POD) 1, 3, 7, and 28, while postoperative CVP was obtained on POD 1 and 3 , as well as POD 7 when possible. All data were obtained from a review of hospital records of patients who provided informed consent.

\section{Operative Procedures}

Implantation was performed either through a median sternotomy or as previously described. ${ }^{19,20}$ We prefer performing all of the related procedures without using cardioplegic arrest, unless the LV is seriously damaged by acute myocardial infarction or an LV thrombus is identified. If RVAD was needed due to difficulty with weaning from cardiopulmonary bypass, we used extracorporeal membranous oxygenation (ECMO) established with right atrium and pulmonary artery cannulation. If right heart failure was so severe that the surgeon considered that weaning from the RVAD with ECMO was impossible within a few weeks after surgery, RVAD with an implantable device was utilized instead of ECMO.

\section{Statistical Analysis}

Data were analyzed using Statview 5.0 (SAS Institute, Cary, NC, USA) and JMP 7.0 (SAS Institute). Results are expressed as mean \pm SD. Mann-Whitney U-test was used for comparison of continuous variables and Fisher's exact test was used to compare frequencies between the groups. Spearman's correlation coefficient by rank was calculated for linear correlation analysis between LS and the various parameters. Receiver operator characteristic (ROC) curves and the corresponding area under the curve (AUC) were used to obtain cut-offs for the outcomes. Logistic regression test was used for univariate and multivariate analysis. $\mathrm{P}<0.1$ was used to select variables for the multivariate risk factor MAE analysis. $\mathrm{P}<0.05$ was considered statistically significant.

\section{Results}

\section{Clinical Outcome}

Four patients required RVAD support and were classified as 

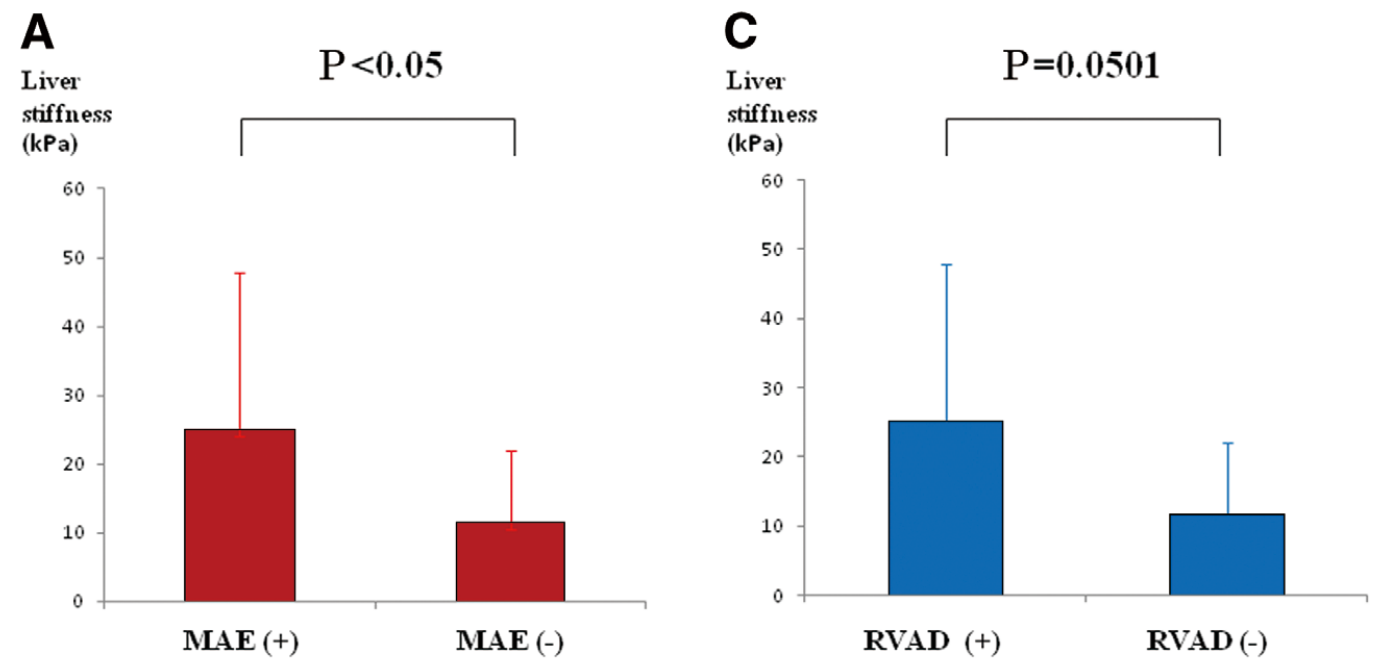

B

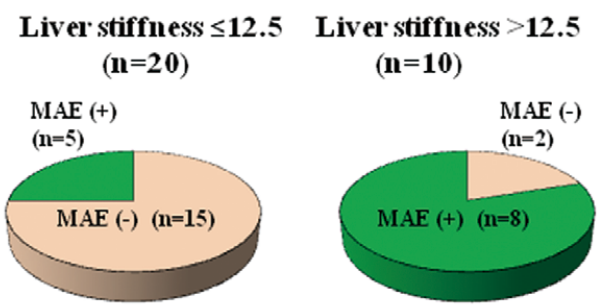

D

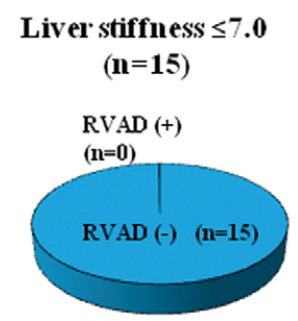

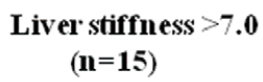

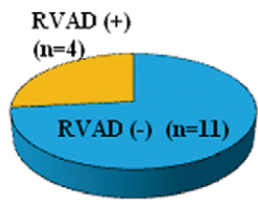

Figure 3. Liver stiffness (LS) vs. presence of (A) major adverse events (MAE); and (C) need for right ventricular assist device (RVAD). (B,D) Use of LS cut-offs for (B) MAE and (D) need for RVAD. (A) LS was significantly higher in patients with MAE than in those without $(22.4 \pm 17.4$ vs. $8.0 \pm 5.0 \mathrm{kPa}, \mathrm{P}<0.05)$. (B) Eighty percent of patients with $\mathrm{LS}>12.5 \mathrm{kPa}$ had MAE while only $25 \%$ of those with LS $\leq 12.5 \mathrm{kPa}$ had them. (C) LS tended to be higher in patients who required RVAD as compared to those without (25.1 \pm 22.7 vs. $11.5 \pm 10.5 \mathrm{kPa}, P=0.051$ ). (D) Among patients with $L S>7.0 \mathrm{kPa}, 27 \%$ required $\mathrm{RVAD}$, while none of the patients with LS $\leq 7.0 \mathrm{kPa}$ required RVAD.

the RVAD group. There was no 30-day mortality in this cohort, with overall in-hospital mortality $10 \%(n=3)$. The cause of death was massive cerebral bleeding in 2 patients and multiple organ failure in 1 . In addition, 2 patients underwent reoperation for bleeding and 1 patient had a deep sternal infection. Five patients had cerebral complications, including 3 with cerebral infarction, with subarachnoid hemorrhage, and 1 with cerebral bleeding. These 13 patients were classified as the MAE group. Ten patients had maximum T-bil $>5.0 \mathrm{mg} / \mathrm{dl}$, and all of them recovered from the liver dysfunction.

\section{Transient Elastography}

Successful measurements were obtained in all patients. Mean preoperative LS in the entire group was $13.3 \pm 13.0 \mathrm{kPa}$, and 23 patients $(77 \%)$ of the entire cohort had abnormal preoperative LS (normal, $<5.5 \mathrm{kPa}$ ). We noted 2 types of perioperative change in regard to LS. On ROC analysis, $\mathrm{LS}=7.0 \mathrm{kPa}$ was identified as a cut-off for RVAD requirement. Therefore, we checked perioperative change of LS in 2 different groups. In patients with LS $\leq 7.0 \mathrm{kPa}$, LS was significantly increased on POD 3 and remained slightly elevated on POD 7 as compared to the preoperative level, while it returned to nearly normal on POD 28 (Figure 1A). In contrast, in patients with LS $>7.0 \mathrm{kPa}$, LS gradually decreased during the postoperative period and still remained higher than normal on POD 28 (Figure 1B). Preop- erative CVP was also higher than that on POD 3. There were no significant differences regarding the amount of diuretics and requirement of hemodialysis between these 2 groups.

\section{LS and Perioperative Factors}

Preoperative CVP had a significant correlation with preoperative LS ( $r=0.515, n=30, P<0.01$; Figure 2A), and preoperative LS was also significantly correlated with CVP on POD 3 ( $r=0.416$, $\mathrm{n}=30, \mathrm{P}<0.05$; Figure $2 \mathrm{~B}$ ). The correlation between preoperative LS and LVEF was not significant. There were no significant relationships between preoperative LS and liver function parameters such as AST, ALT, and T-bil, whereas LS was correlated with preoperative BNP and CRP (Figures 2C,D).

\section{LS and Outcome}

LS was significantly greater in patients with MAE than in those without $(22.4 \pm 17.4$ vs. $8.0 \pm 5.0 \mathrm{kPa}, \mathrm{P}<0.05$; Figure $3 \mathrm{~A})$. On ROC analysis, the cut-off for LS was $12.5 \mathrm{kPa}$ (AUC, 0.82). Among patients with $\mathrm{LS}>12.5 \mathrm{kPa}, 80 \%$ had MAE, while only $25 \%$ of those with $\mathrm{LS} \leq 12.5 \mathrm{kPa}$ had MAE. There were also no deaths among patients with $\mathrm{LS} \leq 12.5 \mathrm{kPa}$ (Figure 3B). Finally, preoperative LS between patients with and without MAE was significant on univariate analysis, and on multivariate analysis only preoperative LS was identified as a risk factor for MAE (Table 2). 


\begin{tabular}{|c|c|c|c|}
\hline & OR & $95 \% \mathrm{Cl}$ & P-value \\
\hline \multicolumn{4}{|l|}{ Univariate analysis } \\
\hline Age (years) & 1.047 & $0.983-1.114$ & 0.1512 \\
\hline Gender (M/F) & 1.231 & $0.238-6.359$ & 0.8043 \\
\hline Body weight (kg) & 1.017 & $0.895-1.156$ & 0.7906 \\
\hline Liver stiffness (kPa) & 1.156 & $1.018-1.313$ & 0.0254 \\
\hline INTERMACS profile & 0.913 & $0.311-2.685$ & 0.8693 \\
\hline Preoperative T-bil (mg/dl) & 0.953 & $0.637-1.425$ & 0.8154 \\
\hline Preoperative AST (IU/L) & 0.988 & $0.957-1.021$ & 0.4751 \\
\hline Preoperative ALT (IU/L) & 0.992 & $0.973-1.011$ & 0.4156 \\
\hline Preoperative $\mathrm{Cr}$ (mg/dl) & 1.813 & $0.657-5.011$ & 0.2502 \\
\hline Preoperative CRP (mg/dl) & 1.045 & $0.826-1.323$ & 0.7123 \\
\hline Preoperative BNP (pg/ml) & 1.000 & $0.999-1.001$ & 0.8885 \\
\hline $\operatorname{LVEF}(\%)$ & 1.099 & $0.979-1.234$ & 0.1084 \\
\hline Preoperative sPAP $(\mathrm{mmHg})$ & 1.058 & $0.972-1.153$ & 0.1924 \\
\hline Preoperative PCWP $(\mathrm{mmHg})$ & 1.081 & $0.962-1.216$ & 0.1899 \\
\hline Preoperative CVP (mmHg) & 1.175 & $0.979-1.411$ & 0.0832 \\
\hline \multicolumn{4}{|l|}{ Multivariate analysis } \\
\hline Liver stiffness (kPa) & 1.145 & $1.005-1.305$ & 0.0402 \\
\hline Preoperative CVP $(\mathrm{mmHg})$ & 1.089 & $0.942-1.259$ & 0.2508 \\
\hline
\end{tabular}

Abbreviations as in Table 1.

As for the incidence of RVAD, LS tended to be higher in patients who required RVAD as compared to those without (25.1 \pm 22.7 vs. $11.5 \pm 10.5 \mathrm{kPa}, \mathrm{P}=0.051$; Figure $3 \mathrm{C})$. On ROC analysis, $7.0 \mathrm{kPa}$ was the cut-off for LS (AUC, 0.87). Among patients with $\mathrm{LS}>7.0 \mathrm{kPa}, 27 \%$ required $\mathrm{RVAD}$, while none of the patients with $\mathrm{LS} \leq 7.0 \mathrm{kPa}$ required RVAD (Figure 3D). We also examined the relationship between CVP/PCWP and RVAD requirement. There was no significant difference between them.

\section{Discussion}

In the present study there was significant increase in LS in patients with severe heart failure and without known pre-existing liver disease, given that mean LS reached $13.3 \mathrm{kPa}$ in those patients, which is substantially higher than normal. In particular, $15(50 \%)$ of 30 patients had LS above the threshold normally used to diagnose substantial fibrosis, while $10(33 \%)$ had LS at a level that usually indicates a diagnosis of cirrhosis. We also found that longitudinal changes in LS occurred in parallel with perioperative changes in volume status in patients with LS $\leq 7.0 \mathrm{kPa}$. In addition, as well as the correlations of LS with preoperative $\mathrm{BNP}$ and with $\mathrm{CRP}$, there was also a correlation between LS and CVP prior to operation, as well as on POD 3. Finally, we showed that preoperative LS may be a possible predictor of right heart support during operation, as well as of postoperative outcome.

To the best of our knowledge, this is the first study to prospectively assess the performance of LS for predicting clinical outcome and complications in comparison with various hemodynamic parameters. The present findings may have an impact on postoperative management for severe heart failure patients, because this novel modality is non-invasive and user friendly, while it also provides immediate results and good reproducibility. ${ }^{13,14}$

There are 2 possible reasons to explain high preoperative LS: the direct effect of CVP and the impact of chronic heart disease on the liver. A previous study using an animal model showed that CVP directly controls LS in a reversible manner, and that $\mathrm{LS}$ is a linear function of intravenous pressure, reaching the upper detection limit of $75 \mathrm{kPa}$ at an intravenous pressure of $36 \mathrm{cmH}_{2} \mathrm{O} .{ }^{17}$ Generally, patients who require LVAD implantation have both right and left heart failure symptoms, ${ }^{21}$ thus most have relatively high CVP. Several reports have noted that heart failure plays a role in LS determinants. ${ }^{17,22,23}$ Cardiac hepatopathy is thought to be related to hepatic venous congestion, and all of the present patients had a long history of heart failure. Therefore, it is possible that their high LS simply reflected chronic hepatic injury due to cardiac dysfunction, and that LS may reflect acute volume status as well as the chronic effect of heart failure on the liver. Taniguchi et al reported a strong correlation between right atrium pressure and LS, ${ }^{24}$ while in the present study there was a relatively slight correlation between them. This difference might be due to severity, given that all of the present patients had severe heart failure and other complications such as inflammation and chronic liver congestion, which are considered to affect LS.

Perioperative change is another important finding, in that LS showed a shift in volume status during the postoperative period. We found 2 patterns of preoperative LS change. In patients with approximately normal LS, LS increased to a maximum on POD 3, then gradually decreased and reached the preoperative level after 1 month. This change occurred in parallel with the usual postoperative change in fluid status. Following the operation, fluid level reached maximum during the first few days, followed by a depletion phase due to diuretics. Therefore, LS may be useful to evaluate volume status in a non-invasive manner. In patients with high LS, LS had a gradual decrease, although it remained higher than normal after 1 month, which likely reflected perioperative status severity and liver dysfunction. Among patients with preoperative LS $>7.0 \mathrm{kPa}$, most had very high $\mathrm{LS}>10$ or $20 \mathrm{kPa}$, which is higher than the maximum LS in patients with preoperative $\mathrm{LS} \leq 7.0 \mathrm{kPa}$. We assume that this continuous decline in patients with preoperative LS 
$>7.0 \mathrm{kPa}$ reflects continuous unloading of liver congestion. We think that LS may be more useful in patients without liver dysfunction due to heart failure.

We also found a significant correlation between LS and preoperative CVP, as well as with POD 3 CVP. This result is similar to that in previous reports showing that LS is directly influenced by CVP. ${ }^{14}$ Although previous reports noted a specific correlation between CVP and LS, ${ }^{17,24}$ it might not be suitable to compare results from patients with a relatively lower disease level with that of the present cohort.

Interestingly, even though LS did not have a significant correlation with PAP, PCWP, or LVEF, which is similar to other results, there was a significant correlation between LS and POD 3 CVP. A possible explanation is that LS may reflect right heart function. ${ }^{22}$ Following LVAD implantation, cardiac return generally increases, while right ventricle load also increases. Given that LS is also determined by already established liver fibrosis secondary to chronic hepatic congestion, ${ }^{25}$ higher LS indicates long-term liver damage due to right heart failure. This chronic change, as well as the direct influence by CVP, may be determinants of LS. We also found a significant relationship between preoperative LS and BNP, which is in contrast to Millonig et al, ${ }^{17}$ but agrees with Hopper et al. ${ }^{22}$ This discrepancy may be related to the low number of subjects in each study. Although it is possible that LS is directly correlated with heart failure, further study is needed to clarify the direct relationship with BNP.

The present investigation provides several important findings. LS may be effective for prediction of surgical outcome. Transient elastography is a rapid and non-invasive means of assessing LS, and can be readily incorporated into clinical evaluation of patients with heart failure to assist with their management. We found that LS might also be useful to predict the need for additional right heart support such as RVAD in patients undergoing LVAD implantation. Notably, all patients with RVAD had high LS, thus care must be taken when treating patients with elevated LS. Furthermore, LS might be useful to predict requirement of TAP. A previous study found a significant relationship between liver function and severity of tricuspid regurgitation, in that severe regurgitation causes severe hepatic congestion. ${ }^{26}$ Also, LS might be a possible risk factor for postoperative outcome, which is compatible with a previous study that showed that intensive care patients with LS in the upper quartile had increased short-term mortality. ${ }^{27}$ In addition, several reports have described the relationship between liver dysfunction and outcome. ${ }^{2-4}$ Also, LS may be a predictor of recovery from liver dysfunction. In the present study, however, we were not able to investigate that possibility, because all of the present patients recovered from liver dysfunction. The prognostic importance of LS may be a reflection of poor hemodynamic status and underlying secondary hepatic injury. Based on these findings, LS might be useful as a screening test for clinical outcome in patients scheduled for LVAD surgery. Some reports have described the importance of CVP/ PCWP as a predictor of RVAD in patients undergoing LVAD implantation. ${ }^{28}$ In the present study, we did not find a relationship between CVP/PCWP and RVAD requirement, which may have been due to the small number of patients who required RVAD.

\section{Study Limitations}

There were several limitations in this study. No liver biopsy results were available, which would have allowed a better description of the relationship between LS and histologic findings. Therefore, we were unable to correlate LS with the un- derlying histological severity of liver fibrosis. The invasive procedure needed to obtain such results, however, was considered inappropriate and therefore unethical. Furthermore, the characteristics of the present cohort limited interpretation of the findings. Patients requiring LVAD implantation are categorized as critically ill. To prove the usefulness of LS in general heart failure patients, an investigation of such a cohort is needed. It is also important to keep in mind that LS is influenced by several specific conditions, such as hepatitis, ${ }^{13}$ inflammation, ${ }^{29}$ and cholestasis. ${ }^{30}$ Therefore, even though we excluded patients with hepatitis and obvious previous liver disease, the present results must be interpreted with caution in regard to those conditions. Finally, the sample size was not large enough for definitive conclusions to be drawn. Although further multicenter studies with larger groups of patients are necessary to confirm the present results, the present findings highlight the novel concept that LS can be useful for management of severe heart failure patients.

\section{Conclusions}

LS was correlated with preoperative severity in patients with severe heart failure, and may be useful to predict the requirement of additional right heart support or postoperative complications in patients undergoing LVAD implantation, while its change reflected liver congestion. This novel modality may be useful for non-invasive assessment of patients with severe heart failure.

\section{Disclosures}

None.

\section{Source of Funding}

None.

\section{References}

1. Richman SM, Delman AJ, Grob D. Alterations in indices of liver function in congestive heart failure with particular reference to serum enzymes. Am J Med 1961; 30: 211-225.

2. Kubo SH, Walter BA, John DH, Clark M, Cody RJ. Liver function abnormalities in chronic heart failure: Influence of systemic hemodynamics. Arch Intern Med 1987; 147: 1227-1230.

3. Allen LA, Felker GM, Pocock S, McMurray JJ, Pfeffer MA, Swedberg $\mathrm{K}$, et al. Liver function abnormalities and outcome in patients with chronic heart failure: Data from the Candesartan in Heart Failure: Assessment of Reduction in Mortality and Morbidity (CHARM) program. Eur J Heart Fail 2009; 11: 170-177.

4. Poelzl G, Ess M, Mussner-Seeber C, Pachinger O, Frick M, Ulmer H. Liver dysfunction in chronic heart failure: Prevalence, characteristics and prognostic significance. Eur J Clin Invest 2012; 42: 153 163.

5. van Deursen VM, Damman K, Hillege HL, van Beek AP, van Veldhuisen DJ, Voors AA. Abnormal liver function in relation to hemodynamic profile in heart failure patients. J Card Fail 2010; 16: 84-90.

6. Fujita Y, Fujino Y, Matsumiya G, Sawa Y, Mashimo T, Matsuda H, et al. Postoperative hyperbilirubinemia after implantation of left ventricular assist device is associated with poor postoperative liver perfusion. J Artif Organs 2005; 8: 28-33.

7. Saito S, Matsumiya G, Sakaguchi T, Miyagawa S, Yoshikawa Y, Yamauchi T, et al. Risk factor analysis of long-term support with left ventricular assist system. Circ J 2010; 74: 715-722.

8. Masai T, Sawa Y, Ohtake S, Nishida T, Nishimura M, Fukushima N, et al. Hepatic dysfunction after left ventricular mechanical assist in patients with end-stage heart failure: Role of inflammatory response and hepatic microcirculation. Ann Thorac Surg 2002; 73: 549-555.

9. Imamura T, Kinugawa K, Shiga T, Endo M, Kato N, Inaba T, et al. Preoperative levels of bilirubin or creatinine adjusted by age can predict their reversibility after implantation of left ventricular assist device. Circ J 2013; 77: 96-104.

10. Fukushima N. How can we predict reversibility of organ dysfunction 
after implantation of left ventricular assist device? Circ J 2013; 77: $45-46$.

11. Yoshioka D, Sakaguchi T, Saito S, Miyagawa S, Nishi H, Yoshikawa $\mathrm{Y}$, et al. Predictor of early mortality for severe heart failure patients with left ventricular assist device implantation: Significance of INTERMACS level and renal function. Circ J 2012; 76: 1631-1638.

12. Nishi H, Sakaguchi T, Miyagawa S, Yoshikawa Y, Fukushima S, Saito $S$, et al. Frequency, risk factors and prognosis of postoperative hyperbilirubinemia after heart valve surgery. Cardiology 2012; 122: $12-19$.

13. Castéra L, Vergniol J, Foucher J, Le Bail B, Chanteloup E, Haaser $\mathrm{M}$, et al. Prospective comparison of transient elastography, Fibrotest, APRI, and liver biopsy for the assessment of fibrosis in chronic hepatitis C. Gastroenterology 2005; 128: 343-350.

14. Martínez SM, Crespo G, Navasa M, Forns X. Noninvasive assessment of liver fibrosis. Hepatology 2011; 53: 325-335.

15. Alisi A, Pinzani M, Nobili V. Diagnostic power of fibroscan in predicting liver fibrosis in nonalcoholic fatty liver disease. Hepatology 2009; 50: 2048-2049.

16. Colli A, Pozzoni P, Berzuini A, Gerosa A, Canovi C, Molteni EE, et al. Decompensated chronic heart failure: Increased liver stiffness measured by means of transient elastography. Radiology 2010; 257: 872-878.

17. Millonig G, Friedrich S, Adolf S, Fonouni H, Golriz M, Mehrabi A, et al. Liver stiffness is directly influenced by central venous pressure. J Hepatol 2010; 52: 206-210.

18. Fourquet B, Hasquenoph JM, Yon S, Fournier C, Mal F, Christidis $\mathrm{C}$, et al. Transient elastography: A new noninvasive method for assessment of hepatic fibrosis. Ultrasound Med Biol 2003; 29: 1705-1713.

19. Sakaguchi T, Matsumiya G, Yoshioka D, Miyagawa S, Nishi H, Yoshikawa Y, et al. DuraHeart ${ }^{\mathrm{TM}}$ magnetically levitated left ventricular assist device: Osaka University experience. Circ J 2013; 77: $1736-1741$.

20. Aaronson KD, Slaughter MS, Miller LW, McGee EC, Cotts WG, Acker MA, et al. Use of an intrapericardial, continuous-flow, centrifu- gal pump in patients awaiting heart transplantation. Circulation 2012; 125: $3191-3200$.

21. Craig ML. Management of right ventricular failure in the era of ventricular assist device therapy. Curr Heart Fail Rep 2011; 8: 65-71.

22. Hopper I, Kemp W, Porapakkham P, Sata Y, Condon E, Skiba M, et al. Impact of heart failure and changes to volume status on liver stiffness: Non-invasive assessment using transient elastography. Eur $J$ Heart Fail 2012; 14: 621-627.

23. Alegre F, Herrero JI, Iñarrairaegui M, Gavira JJ, Pujol C, Montero $\mathrm{A}$, et al. Increased liver stiffness values in patients with heart failure. Acta Gastroenterol Belg 2013; 76: 246-250.

24. Taniguchi T, Sakata Y, Ohtani T, Mizote I, Takeda Y, Asano Y, et al. Usefulness of transient elastography for noninvasive and reliable estimation of right-sided filling pressure in heart failure. Am J Cardiol 2014; 76: 552-558.

25. Friedrich-Rust M, Koch C, Rentzsch A, Sarrazin C, Schwarz P, Herrmann E, et al. Noninvasive assessment of liver fibrosis in patients with Fontan circulation using transient elastography and biochemical fibrosis markers. J Thorac Cardiovasc Surg 2008; 135: 560-567.

26. Lau GT, Tan HC, Kritharides L. Type of liver dysfunction in heart failure and its relation to the severity of tricuspid regurgitation. $\mathrm{Am}$ J Cardiol 2002; 90: 1405-1409.

27. Lindvig K, Mössner BK, Pedersen C, Lillevang ST, Christensen PB Liver stiffness and 30-day mortality in a cohort of patients admitted to hospital. Eur J Clin Invest 2012; 42: 146-152.

28. Shiga T, Kinugawa K, Imamura T, Kato N, Endo M, Inaba T, et al. Combination evaluation of preoperative risk indices predicts requirement of biventricular assist device. Circ J 2012; 76: 2785-2791.

29. Arena U, Vizzutti F, Corti G, Ambu S, Stasi C, Bresci S, et al. Acute viral hepatitis increases liver stiffness values measured by transient elastography. Hepatology 2008; 47: 380-384.

30. Millonig G, Reimann FM, Friedrich S, Fonouni H, Mehrabi A, Büchler MW, et al. Extrahepatic cholestasis increases liver stiffness (FibroScan) irrespective of fibrosis. Hepatology 2008; 48: 1718-1723. 